\title{
Alfabetización en salud: concepto y dimensiones. Proyecto europeo de alfabetización en salud
}

\section{Health Literacy: Concept and Dimensions. European Project of Health Literacy}

\author{
María Falcón Romero \\ Aurelio Luna Ruiz-Cabello \\ HLS-EU Consorcio. Universidad de Murcia \\ (Murcia, España)
}

\begin{abstract}
más participantes en el Consorcio Europeo de Alfabetización en Salud
Universidad de Maastricht, Paises Bajos: H Brand, S van den Broucke y K Sørensen. National School of Public Health, Grecia: D Agrafiodis, E loannidis and B Kondilis. University College of Dublin, National University of Ireland: G Doyle, J Fullam y K Cafferkey.

Ludwig Boltzmann Gesellschaft GmbH, Austria: J Pelikan y F Röethlin. Instytut Kardiologii, Polonia: Z Slonska.

University of Murcia, España: M Falcón, MD Perez-Carceles, JJ Lopez y A Luna.

Medical University-Sofia, Bulgaria: Ka Tchamov y A Zhekov.

National Institute of Public Health and the Environment, Holanda: M Droomers, J Schuit, I van der Heide and E Uiters.

NRW Centre for Health, Alemania: G Ward and M Mensing
\end{abstract}

\section{Cómo citar el artículo}

Falcón, M. y Luna, A. (2012). Alfabetización en salud: concepto y dimensiones. Proyecto europeo de alfabetización en salud. Revista de Comunicación y Salud, 2(2), pp. 91-98. DOI: http://doi.org/10.35669/revistadecomunicacionysalud.2012.2(2).91-98 
La alfabetización en salud (AES), traducción del termino inglés health literacy, es un concepto complejo introducido en los 70 en el mundo anglosajón, que cada día va ganando mas reconocimiento entre la comunidad científica internacional. Existen multitud de definiciones del término en inglés y diversas traducciones al castellano de health literacy. Entre las más conocidas y utilizadas se encuentra la que recoge la OMS en su glosario de términos de salud pública que la define como "las habilidades sociales y cognitivas que determinan el nivel de motivación y la capacidad de una persona para acceder, entender y utilizar la información de forma que le permita promover y mantener una buena salud". Sin embargo, a pesar de la unanimidad sobre su importancia, no hay una definición unánimemente aceptada del concepto de alfabetización en salud, y sus dimensiones constitutivas siguen siendo objeto de controversia, así como tampoco hay un consenso sobre qué instrumento es el más adecuado para medirla (Sørensen et al., 2011; Baker, 2006).

En casi todas las facetas de la vida las personas nos enfrentamos a situaciones y decisiones que afectan a nuestra salud. Hoy día tenemos a nuestro alcance más información que nunca para lograr llevar una vida sana, adquirir estilos de vida saludables, adoptar acciones que nos ayuden a prevenir las enfermedades o para involucrarnos activamente en los tratamientos de las enfermedades que padecemos, pero ¿estamos preparados para acceder a esta información, entenderla, procesarla y aplicarla adecuadamente a nuestras circunstancias particulares?

La AES va mas allá de la alfabetización general, significa no solo saber leer el prospecto de un medicamento o entender la información que nos facilita el profesional sanitario respecto a un tratamiento, sino que implica saber como acceder a la información adecuada, interpretarla, juzgarla y aprovecharla para tomar decisiones bien fundamentadas sobre nuestra propia salud y la de nuestra comunidad.

Es imprescindible no olvidar un aspecto esencial respecto a la alfabetización en salud; ésta no depende exclusivamente de las capacidades individuales de los ciudadanos, hay que contextualizarla en un escenario concreto, pues resulta de la interacción entre las demandas de los diferentes sistemas de salud y las habilidades de los individuos inmersos en ellos.

Según los expertos en salud pública, un individuo con un nivel adecuado de AES tiene las competencias necesarias para gestionar su salud de una forma responsable (Kickbusch, 2008). En cierto modo se trata de una exigencia cívica, en la medida en que en la actualidad la mayoría de los países desarrollados tiene unos sistemas de salud diseñados para ciudadanos motivados, competentes y responsables; y es, a su vez, una exigencia ética en la medida en que el cuidado de uno mismo, y por consiguiente, el velar por la propia salud es una obligación moral fundamental en una sociedad que consume cada vez más recursos sanitarios en un escenario de limitación de los mismos.

Muchos de los trabajos de investigación en el campo de la AES han estudiado cómo ésta influye en la salud de los individuos, los grupos de riesgo más vulnerables y el coste económico que supone una alfabetización en salud pobre. La alfabetización en salud tiene un impacto directo y significativo sobre la salud individual y pública que queda patente especialmente en los colectivos más vulnerables (ancianos, polimedicados, enfermos crónicos...) y que se ha 
relacionado con un entendimiento problemático de información de salud e instrucciones médicas (Cho Yi et al., 2008; Davis et al., 2004; Gazmararian et al., 2003 y Parker et al., 2003), una inadecuada autogestión de la enfermedad (Gazmararian et al., 2003), un uso limitado de los recursos preventivos (Baker et al., 2004; Lindau et al., 2002; Lindau et al., 2006; Scott et al., 2002; Rogers et al., 2006; von Wagner et al., 2007), un aumento de las hospitalizaciones y de los costes sanitarios (Baker et al., 2002; Howard et al., 2006) y con un incremento de las tasas de mortalidad (Sudore et al., 2006).

Todas estas consecuencias conllevan un coste social y económico significativo. Para hacernos una idea, la National Academy on an Aging Society de Estados Unidos estima que una alfabetización en salud baja supone un coste adicional de 73 billones de dólares al año en los fondos que este país destina a su sistema de salud. Es más que patente la conveniencia de que los distintos países desarrollen instrumentos para medir de forma periódica la alfabetización en salud de su población y pongan en marcha iniciativas efectivas para aumentar los niveles partiendo de datos objetivos, comparables y reproducibles.

\section{Proyecto europeo de alfabetización en salud}

Conscientes de las lagunas de investigación en el campo de la alfabetización en salud, sobre todo en Europa, el proyecto (HLS-EU) financiado por la Comisión Europea y formado por 8 países (Grecia, Irlanda, los Países Bajos, Austria, Polonia, España, Bulgaria y Alemania) se planteó la necesidad de documentar, por primera vez, datos sobre la AES en los países europeos y, así, contar con indicadores para una vigilancia tanto a nivel nacional como de toda la UE y establecer una Red Europea de Alfabetización en Salud.

La primera etapa del proyecto consistió en realizar una revisión sistemática de la bibliografía especializada con el fin de identificar las definiciones, los marcos conceptuales y las herramientas de medida existentes de alfabetización en salud. Se llevó a cabo un análisis del contenido para identificar las dimensiones centrales de la alfabetización en salud y desarrollar un modelo integrado de medida.

Basándose en esta revisión, el proyecto de la HLS-EU propone su propio modelo, que abarca tanto el punto de vista médico-asistencial como el de salud pública de la alfabetización en salud.

La definición que se propone es: "La alfabetización en salud se basa en la alfabetización general y engloba las motivaciones, los conocimientos y las competencias de las personas para acceder, entender, evaluar y aplicar la información sobre la salud en la toma de decisiones

1. Disponible en http://www.agingsociety/publications/fact/fact_low.html 
sobre la atención y el cuidado sanitario, la prevención de enfermedades y la promoción de la salud para mantener y mejorar la calidad de vida a lo largo de ésta"(Sørensen et al., 2011).

La combinación de las cuatro dimensiones, referidas al procesamiento de la información sobre la salud (acceder, entender, evaluar y aplicar) con los tres niveles de dominio (atención y cuidado del enfermo, prevención y promoción de la salud), produce una matriz con 12 dimensiones.

Tabla 1. Dimensiones de la AES según la HLS-EU

\begin{tabular}{|l|l|l|l|l|}
\hline Health literacy & Acceder/obtener & Entender & Procesar/juzgar & Aplicar/usar \\
\hline $\begin{array}{l}\text { Atención y } \\
\text { Habidado. } \\
\text { para : }\end{array}$ & $\begin{array}{l}\text { 1) acceder a } \\
\text { información } \\
\text { medica o clínica }\end{array}$ & $\begin{array}{l}\text { 2) entender la } \\
\text { información medica } \\
\text { y su significado }\end{array}$ & $\begin{array}{l}\text { 3) interpretar y evaluar la } \\
\text { información médica }\end{array}$ & $\begin{array}{l}\text { 4) tomar decisiones } \\
\text { informadas en temas } \\
\text { médicos }\end{array}$ \\
\hline $\begin{array}{l}\text { Prevención de } \\
\text { enfermedades. }\end{array}$ & $\begin{array}{l}\text { 5) acceder a } \\
\text { información } \\
\text { Habilidad } \\
\text { para : }\end{array}$ & $\begin{array}{l}\text { 5) entender la } \\
\text { riesgo para la } \\
\text { información sobre } \\
\text { factores de riesgo y }\end{array}$ & $\begin{array}{l}\text { 7) interpretar y evaluar la } \\
\text { información sobre factores de } \\
\text { riesgo para la salud }\end{array}$ & $\begin{array}{l}\text { 8) tomar decisiones } \\
\text { informadas sobre } \\
\text { factores de riesgo }\end{array}$ \\
\hline $\begin{array}{l}\text { Promoción de } \\
\text { la salud. } \\
\text { Habilidad } \\
\text { para : }\end{array}$ & $\begin{array}{l}\text { 9) ponerse al dia } \\
\text { sobre los } \\
\text { determinantes de } \\
\text { la salud }\end{array}$ & $\begin{array}{l}\text { 10) entender la } \\
\text { información sobre } \\
\text { los determinantes } \\
\text { de la salud y su } \\
\text { significado }\end{array}$ & $\begin{array}{l}\text { 11) interpretar y evaluar la } \\
\text { información sobre los } \\
\text { determinantes de la salud }\end{array}$ & $\begin{array}{l}\text { 12) tomar decisiones } \\
\text { informadas sobre } \\
\text { determinantes de la } \\
\text { salud }\end{array}$ \\
\hline
\end{tabular}

Fuente: Sørensen (2011)

A partir de aquí se diseñó el cuestionario (HLS-EU) que incluye entre 3 y 5 items específicamente formulados por cada una de estas subdimensiones, que resultan en un total de 47 cuestiones. Estos ítems indagan sobre determinadas situaciones o tareas en la que se necesita gestionar información relevante para la salud en aspectos relacionados con la asistencia sanitaria, la prevención de enfermedades y la promoción de la salud. Las respuestas clasifican el grado de dificultad percibida por el encuestado en cada tarea o situación, baremándolas en una escala de Likert con 4 categorías (muy fácil, fácil, difícil, muy difícil).

Algunos ejemplos de los ítems recogidos en el cuestionario son:

En una escala de "muy fácil" a "muy difícil", indique cuál es el grado de dificultad que encontraría para realizar las siguientes actividades:

$5 . .$. entender lo que le dice el médico.

$12 . .$. juzgar si la información que aparece en los medios de comunicación sobre una enfermedad es fiable (TV, Internet u otros medios de información). 
18... encontrar información sobre la manera de abordar problemas de salud mental como el estrés o la depresión.

$38 . .$. comprender la información incluida en los envases de alimentos.

$47 . .$. involucrarse en actividades que mejoren la salud y el bienestar de su comunidad.

La encuesta europea sobre alfabetización en salud la realizó TNS Opinion en representación del consorcio HLS-EU aplicando los estándares del Eurobarómetro en sus procedimientos de muestreo y metodología. Para recabar información se empleó la técnica de las encuestas personales asistidas por ordenador (CAPI, por sus siglas en inglés). La muestra incluyó un total de 1.000 encuestados en cada uno de los ocho países socios.

A partir de los resultados obtenidos utilizando las medias de las 47 variables del instrumento del HLS-EU se calculó el índice general de alfabetización en salud (0-50), siendo 0 el valor de una alfabetización en salud mínima y 50 la máxima.

El índice general de alfabetización en salud comprende el ajuste o relación que existe entre las competencias, expectativas y experiencias del individuo con las demandas de su entorno. A su vez, la puntuación alcanzada permite definir 4 niveles de alfabetización en salud:

0-25 Puntos. Alfabetización en Salud INADECUADA.

$>25-33$ Puntos. Alfabetización en Salud PROBLEMÁTICA.

$>33-42$ Puntos. Alfabetización en Salud SUFICIENTE.

>42-50 Puntos. Alfabetización en Salud EXCELENTE.

Los resultados de la HLS-EU no son muy alentadores. La alfabetización en salud del 12,4\% de los europeos resultó inadecuada, mientras que un 35,2\% tenía una alfabetización en salud problemática. En otras palabras, una de cada dos personas en Europa tiene una alfabetización en salud limitada.

Las diferencias porcentuales relacionadas con las limitaciones en alfabetización en salud son considerables entre los países europeos participantes:

Gráfico 1. Porcentajes de alfabetización general en salud

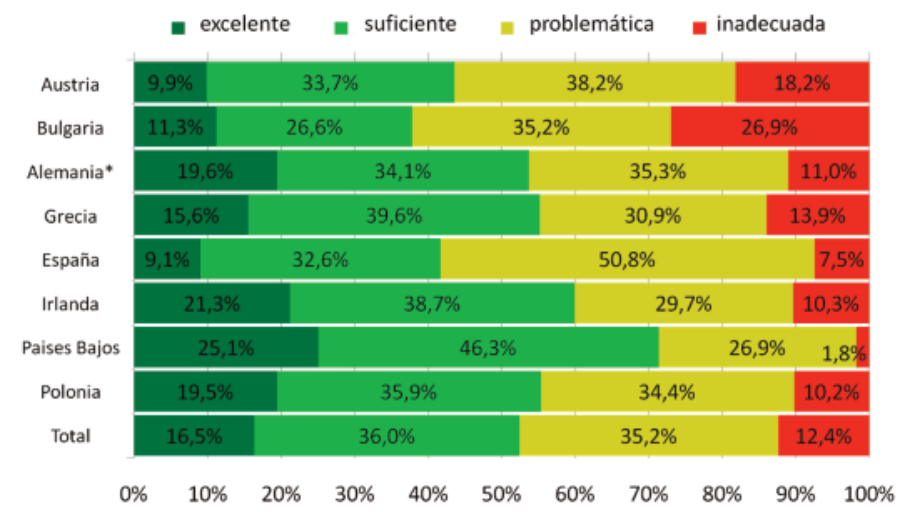

Fuente: Proyecto HLS-EU http://inthealth.eu/research/health-literacy-hls-eu 
Entre los ocho países participantes en el estudio HLS-EU se han detectado diferencias significativas en el nivel y variación de la alfabetización en salud, los gradientes sociales que inciden sobre la alfabetización en salud, así como en el tamaño de los grupos vulnerables cuya alfabetización en salud es limitada. Estas diferencias pueden explicarse, en parte, por la heterogeneidad de la composición socioeconómica y sociodemográfica de las muestras nacionales. Del mismo modo pueden considerarse los efectos de las competencias personales de los encuestados y las diferencias en las altas exigencias de alfabetización en salud en los distintos contextos culturales y sociales, por lo que se necesita un análisis más completo y detallado que permita esclarecer el posible impacto de estos efectos.

Tal y como se muestra en el gráfico 1, la muestra española se caracteriza (positivamente) por un porcentaje bastante reducido de casos con alfabetización inadecuada, que contrasta con un muy elevado porcentaje de casos con alfabetización problemática $(50,8 \%)$. Al comparar los porcentajes de casos con alfabetización en salud limitada entre los distintos países queda patente que las necesidades de alfabetización sanitaria en España deberían priorizar su atención en el bloque etiquetado como problemático. Obsérvese en el gráfico 2 que más de un $25 \%$ de la población en España está ubicado en la zona superior de la alfabetización problemática (en amarillo) con puntuaciones entre 30 y 32; esto supone un gran reto en nuestro país pues un pequeño esfuerzo conseguiría sobre esta zona un gran resultado.

Gráfico 2. Porcentaje de frecuencia del índice general de la puntuación en alfabetización en salud de la población española.

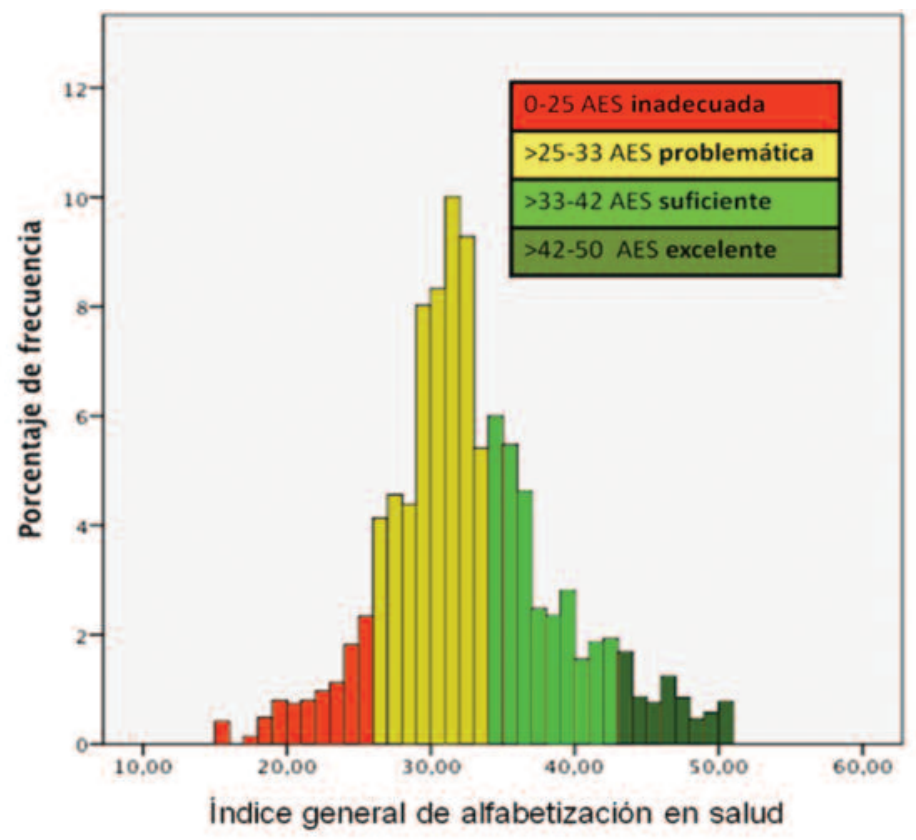

Fuente: Proyecto HLS-EU.

El estudio HLS-EU muestra que en la población encuestada existen correlaciones estadísticamente significativas entre la alfabetización en salud, la salud medida como salud percibida y las características socio-demográficas o socio-económicas de los encuestados en 
función de su edad, situación laboral, nivel de educación formal, carencia de recursos financieros y estado social percibido, de forma que aquellos en situaciones más desfavorables son los que tienen peores niveles de alfabetización en salud.

La mejora de la alfabetización en salud requiere un abordaje multifactorial, pluridisciplinar y multisectorial. Esto supone un compromiso por parte de todos los sectores implicados: el ámbito educativo y sanitario, la industria farmacéutica y de la alimentación, los medios de comunicación científicos y divulgativos, la representación de los colectivos de profesionales sanitarios, pacientes y usuarios, y, por supuesto, de aquellos con responsabilidades políticas. La colaboración y el mutuo asesoramiento son los componentes fundamentales para aumentar la eficacia de las medidas que se adopten, así como para incrementar los niveles de alfabetización en salud de nuestra comunidad. Las intervenciones comunitarias son más eficaces cuando la educación viene acompañada de medidas estructurales que facilitan y refuerzan cambios sostenibles en las conductas; y para ello necesitamos el apoyo de las instituciones y el compromiso político de la administración.

La mejora de la alfabetización en salud debería tratarse como una línea estratégica que actúe sobre la atención y el cuidado, la prevención de enfermedades y la promoción de la salud. Las actuaciones sobre AES serán enfocadas desde dos perspectivas: por una parte, interviniendo sobre pacientes y ciudadanos para aumentar sus competencias y, por otra, simplificando el Sistema de Salud para facilitar su accesibilidad, disminuyendo las exigencias de alfabetización en salud en los distintos contextos culturales y sociales, mejorando la calidad de la información y la comunicación en salud. Es necesario concienciar a los profesionales sanitarios del impacto en la salud individual y colectiva de la AES limitada, y adaptar los esquemas tradicionales de educación en salud para conseguir que la comunicación oral, escrita, electrónica e interpersonal entre los consumidores, pacientes y ciudadanos y los proveedores de salud mejoren.

Más información en: http://inthealth.eu/research/health-literacy-hls-eu/

\section{Referencias}

Baker, D. et al. (2002). Functional Health Literacy and the Risk of Hospital Admission among Medicare Managed Care Enrollees. American Journal of Public Health. Vol. 92, nº 8, pp. 1.2781.283

Baker, D. et al. (2004). Health Literacy and Use of Outpatient Physician Services by Medicare Managed Care Enrollees. Journal of General Internal Medicine. Vol. 19, $n^{\circ} 3$, pp. 215-220.

Baker, D. (2006). The Meaning and the Measure of Health Literacy. Journal of General Internal Medicine. Vol. 21, n 8, pp. 878-883.

Cho, Y.I.; Lee, S.Y.D.; Arozullah, A.M. y Crittenden, K.S. (2008). Effects of Health Literacy on Health Status and Health Service Utilization amongst the Elderly. Social Science \& Medicine. Vol. 66, n 8 , pp. 1.809-1.816. 
Davis, T. y Wolf, M.S. (2004). Health Literacy: Implications for Family Medicine. Family Medicine. Vol. 36, nº 8, pp. 595-598.

Davis, T. et al. (2006). Low Literacy Impairs Comprehension of Prescription Drug Warning Labels. Journal of General Internal Medicine. Vol. 21, n 8, pp. 847-851.

Gazmararian, J. et al. (2003). Health Literacy and Knowledge of Chronic Disease. Patient Education and Counseling. Vol. 51, n 3, pp. 267-275.

Howard, D. et al. (2006). Impact of Health Literacy on Socioeconomic and Racial Differences in Health in an Elderly Population. Journal of General Internal Medicine. Vol. 21, n 8, pp. 857861.

Kickbusch, I. y Maag, D. (2008). Health Literacy. En International Encyclopedia of Public Health. Volumen 3. Pp. 204-211. Kris, H. y Stella, Q. (Editors). Academic Press.

Nielson-Bohlman, L. et al. (2004). Health Literacy: A Prescription to End Confusion. Washington: The National Academies Press.

Lindau, S. et al. (2002). The Association of Health Literacy with Cervical Cancer Prevention Knowledge and Health Behavior in a Multiethnic Cohort of Women. American Journal of Obstetrics and Gynecology. Vol. 186, pp. 938-943.

Lindau, S. et al. (2006). Health Literacy as a Predictor of Follow-Up after an Abnormal Pap Smear: A Prospective Study. Journal of General Internal Medicine. Vol. 21, pp. 829-834.

Parker, R. et al. (2003). Health Literacy: A Policy Challenge for Advancing High-Quality Health Care. Health Affairs. Vol. 22, n 4, p. 147.

Rogers, E. et al. (2006). Misperceptions of Medical Understanding in Low-Literacy Patients: Implications for Cancer Prevention. Cancer Control. Vol. 13, n 3, pp. 225-229.

Simonds, S. K. (1974). Health Education as Social Policy. Health Education Monograph, 2. Pp. 1-25.

Scott, T. et al. (2002). Health Literacy and Preventive Health Care Use among Medicare Enrollees in a Managed Care Organization. Medical Care. Vol. 40, n 5, pp. 395-404.

Sørensen, Kristine et al. (2011). Literature Review of Health Literacy for the Development of a Theoretical Model. HLS-EU Consortium.

Sudore, R. et al. (2006). Limited Literacy and Mortality in the Elderly: the Health, Aging, and Body Composition Study. Journal of General Internal Medicine. Vol. 21, nº 8, pp. 806-812.

von Wagner, C. et al. (2007). Functional Health Literacy and Health-Promoting Behaviour in a National Sample of British Adults. Journal of Epidemiology and Community Health. Vol. 61, $\mathrm{n}^{\circ}$ 12, pp. 1.086-1.090. 